\title{
On the Mickelsson-Faddeev Extension and Unitary Representations
}

\author{
Doug Pickrell \\ Mathematics Department, University of Arizona, Tucson, AZ 85721, USA
}

\begin{abstract}
The Mickelsson-Faddeev extension is a 3-space analogue of a KacMoody group, where the central charge is replaced by a space of functions of the gauge potential. This extension is a pullback of a universal extension, where the gauge potentials are replaced by operators in a Schatten ideal, as in noncommutative differential geometry. Our main result is that the universal extension cannot be faithfully represented by unitary operators on a separable Hilbert space. We also examine potential consequences of the existence of unitary representations for the Mickelsson-Faddeev extension.
\end{abstract}

\section{Section 1. Introduction}

The Mickelsson-Faddeev extension, denoted by $\hat{M}$ in this paper, is a certain distinguished noncentral abelian extension of the Hamiltonian gauge (or equal time current) group $C^{\infty}(X, G)$ :

$$
0 \rightarrow F \rightarrow \widehat{M} \rightarrow C^{\infty}(X, G) \rightarrow 0 .
$$

(see $[\mathrm{Mi} 2$ or $\mathrm{Fr}]$ and the references cited there).

The kernel of the extension, $F$, consists of a certain class of functions of a gauge potential, the class depending on the dimension of $X$, and it arises in the process of regularizing the gauge (or current) operators. An intriguing question is whether $\hat{M}$ can be represented by unitary operators on a Hilbert space. When $X$ is one dimensional the answer is yes, for then $\hat{M}$ is essentially the Kac-Moody extension and regularization amounts to normal ordering. In higher dimensions regularization involves a multiplicative renormalization, and it is not clear whether this is compatible with unitarity (it is possible to construct nonunitary representationssee [Se or MR]).

One objective of this paper is to cast the Mickelsson-Faddeev extension in a form which is amenable to analysis, at least for $X$ of dimension three. In this case we can take $F$ to consist of real valued affine functions modulo a copy of the integers. We can then think of the extension as a two stage process, the first analytical, the second topological. The first stage is a topologically trivial extension

$$
0 \rightarrow V \rightarrow M \rightarrow C^{\infty}(X, G) \rightarrow 0,
$$


where $V$ consists of (very well behaved) linear functions (relative to a choice of basepoint in the space of $G$-connections), i.e. $M$ is defined by a global analytically nontrivial vector space valued cocycle. The extension $\hat{M}$ can then be viewed as a topologically nontrivial central extension of $M$ :

$$
0 \rightarrow \mathbb{T} \rightarrow \hat{M} \rightarrow M \rightarrow 0 .
$$

The main advantage of this formulation is that $V$ is actually a nuclear space so that we can apply "preliminary Mackey analysis." In particular we can conclude that an irreducible unitary representation of $\hat{M}$ corresponds to the following data: an ergodic invariant measure class for the action of $C^{\infty}(X, G)$ on distributional $\underline{g}$-valued one forms, and an associated class of cocycles. The appropriate measure class, if it exists, is presumably represented by "exp $\left(-\int_{X}\left|F_{A}\right|^{2}\right) D A$ ". Thus for $\operatorname{dimn}(X)=3$ (unlike $\operatorname{dimn}(X)=1$ ), a proof of the existence of unitary representations would have profound analytic consequences.

The main objective of the paper is to study a "universal model" of the Mickelsson-Faddeev extension constructed by Mickelsson and Rajeev ([MR]). They constructed an extension of the restricted group $U_{(4)}$ (the automorphism group of an infinite rank Grassmannian) which pulls back to the MickelssonFaddeev extension when $C^{\infty}(X, G)$ is embedded into $U_{(4)}$, as in Connes's theory of noncommutative differential geometry or Pressley and Segal's approach to loop groups $([C, P S])$. The group $U_{(4)}$ itself does not have any interesting unitary representations ([Pi1]). However, the Mickelsson-Rajeev extension of $U_{(4)}$ does have an interesting representation - the question at hand is whether it can be unitarized.

The answer is negative. More precisely we will show that every separable unitary representation must vanish on the topologically nontrivial part of the kernel (a circle). There is a formal argument indicating that any such representation must actually vanish on the entire kernel.

Notation. If $G$ acts on $A$ by automorphisms, the semidirect product will be denoted by $G \tilde{\times} A$, although as a set we will view it as $A \times G$.

\section{Section 2. The Mickelsson-Faddeev Extension}

Let $G$ be a compact Lie group and choose a representation $G \rightarrow U(n)$, to be fixed throughout this section. We will identify $\underline{u}(n)^{\wedge} \cong$ herm $(n)$ via (a multiple of) trace, so that $\underline{g}^{\wedge} \cong \operatorname{herm}(n) / \underline{g}^{\perp}$.

Let $X$ be an oriented compact three manifold. We will identify the space $\mathscr{A}$ of smooth $G$-connections in the trivial bundle $X \times C^{n}$ with $\Lambda^{1} X \otimes \underline{g}$. The action of $C^{\infty}(X, G)$ on $\mathscr{A}$ is given by $g \cdot A=g A g^{-1}+g d g^{-1}$.

The infinitesimal Mickelsson-Faddeev extension is

$$
0 \rightarrow \operatorname{Affine}(\mathscr{A} ; \mathbf{R}) \rightarrow \hat{m} \rightarrow C^{\infty}(X, \underline{g}) \rightarrow 0,
$$

where the bracket is given by

$$
[(\phi, \xi),(\psi, \eta)]=(\xi \cdot \psi-\eta \cdot \phi+\hat{c}(\xi, \eta),[\xi, \eta]),
$$


$\hat{c}(\xi, \eta) \in \operatorname{Affine}(\mathscr{A}, \mathbf{R})$ is given by

$$
\hat{c}(\xi, \eta)(A)=\int_{X} P(A, d \xi, d \eta)
$$

and $P$ is the polarization of $\operatorname{tr}\left(x^{3}\right)$, i.e. $P(x, y, z)=\frac{1}{2} \operatorname{trace}(x y z+x z y)$. For $A, B, C \in \Lambda^{1} X \otimes \underline{g}, P(A, B, C) \in \Lambda^{3} X$ is given by $P(A, B, C)\left(v_{1}, v_{2}, v_{3}\right)=$ $\Sigma(-1)^{\sigma} P\left(A\left(v_{\sigma 1}\right), B\left(v_{\sigma 2}\right), C\left(v_{\sigma 3}\right)\right)$, so that $P$ is skew in the variables $A, B, C$. The cocycle $\hat{c}$ may vanish identically - this is the nonanomalous case in the context of gauge boson/fermion interactions ([Se]).

The cocycle $\hat{c}(\xi, \eta)$ is actually a linear function of $A \in \Lambda^{1} X \otimes \underline{g}$. In terms of the natural pairing of $\Lambda^{1} X \otimes \underline{g}$ and $\Lambda^{2} X \otimes \underline{g}^{\wedge}$, we have

$$
\hat{c}(\xi, \eta)=\{d \xi, d \eta\}=d \xi \wedge d \eta-d \eta \wedge d \xi
$$

(so $\{$,$\} denotes anticommutator). For this reason we will henceforth identify Affine$ $(\mathscr{A} ; \mathbf{R}) \cong \mathbf{R} \oplus \Lambda^{2} X \otimes \underline{g}^{\wedge}$, so that $\underline{\hat{m}}$ is naturally a nuclear Lie algebra.

Now Mickelsson has demonstrated that (for $\hat{c}$ properly normalized) a corresponding group extension can be realized using the Wess-Zumino functional ([Mi1]). For our purposes it is sufficient to exponentiate the quotient $\underline{m}=\underline{\hat{m}} / \mathbf{R}$. Note that for the extension $\underline{m}, C^{\infty}(X, G)$ acts on the kernel $\Lambda^{2} X \otimes \underline{g^{\wedge}}$ simply by conjugation. The group $M$ is constructed explicitly as follows.

As a manifold we take $M=\Lambda^{2} X \otimes \underline{g}^{\wedge} \times C^{\infty}(X, G)$. The multiplication is then defined by

$$
(E, g)(F, h)=\left(E+g F g^{-1}+\omega(g, h), g h\right),
$$

where the group cocycle $\omega$ is given by

$$
2 \omega(g, h)=\left\{(d g) g^{-1}, g(d h) h^{-1} g^{-1}\right\} .
$$

To check that the multiplication is distributive we need

$$
g \omega(h, k) g^{-1}-\omega(g h, k)+\omega(g, h k)-\omega(g, h)=0 .
$$

This follows from the calculations

$$
\begin{aligned}
2 \omega(g h, k) & =g h\left\{h^{-1} g^{-1}(d g) h, d k k^{-1}\right\} h^{-1} g^{-1}+g h\left\{h^{-1} d h, d k k^{-1}\right\} h^{-1} g^{-1} \\
& =\Phi+g 2 \omega(g, k) g^{-1}, \\
2 \omega(g, h k) & =2 \omega(g, h)+\Phi .
\end{aligned}
$$

To check that the Lie algebra of $M$ is $\underline{m}$, we must verify that

$$
d \xi \wedge d \eta-d \eta \wedge d \xi=\left(D_{1} D_{2} \omega\right)(\xi, \eta)-\left(D_{1} D_{2} \omega\right)(\eta, \xi),
$$

where $D_{1} D_{2} \omega$ is the mixed second derivative at the identity. This follows from

$$
\left.\left.\frac{d}{d s}\right|_{s=0} \frac{d}{d t}\right|_{t=0}\left\{\left(d e^{s \xi}\right) e^{-s \xi}, e^{s \xi}\left(d e^{t \eta}\right) e^{-t \eta} e^{-s \xi}\right\}=d \xi \wedge d \eta-d \eta \wedge d \xi .
$$

The main point here is that $M$ is a topologically trivial extension. This is in contrast to any extension $\hat{M} \rightarrow M$ corresponding to $\underline{\hat{m}} \rightarrow \underline{m}$, where the kernel must be a circle. 
Now suppose $\pi$ is an irreducible unitary representation of $M$. We claim that we can find the following kind of realization for $\pi$. Note that $\left(\Lambda^{2} X \otimes \underline{g}^{\wedge}\right)^{\wedge} \cong \mathscr{D} \Lambda^{1} X \otimes \underline{g}$ ( $g$-valued distributional one-forms). We then have

$$
\begin{aligned}
H(\pi) & \cong L^{2}\left(\mathscr{D} \Lambda^{1} X \otimes \underline{g}, v\right) \otimes \mathscr{H}, \\
{[\pi(E, g) \Phi](A) } & =\exp (i\langle A, E\rangle) \delta(g, A) \rho(g, A)^{1 / 2} \Phi\left(g^{-1} A g\right),
\end{aligned}
$$

where $\delta(g, \cdot) \in U(\mathscr{H})$ satisfies the cocycle condition

$$
\delta(g, A) \delta\left(h, g^{-1} A g\right)=\exp (i\langle A, \omega(g, h)\rangle) \delta(g h, A)
$$

for a.e. $A[v]$, for each $g, h \in C^{\infty}(X, G)$. Here $v$ is a quasi-invariant ergodic measure for the conjugation action of $C^{\infty}(X, G)$ on $\mathscr{D} \Lambda^{1} X \otimes \underline{g}$, and $\rho$ is the Radon-Nikodym derivative.

The argument is a standard one. Let $\eta$ denote the restriction of $\pi$ to the normal subgroup $N=\Lambda^{2} X \otimes \underline{g}^{\wedge}$. Viewing $g \in C^{\infty}(X, G)$ as an automorphism of $N$, we have $\eta^{g}=\eta$.

Because $N$ is abelian, $\eta^{\prime}$ (the commutant) is a type I Von Neumann algebra. Thus we can first decompose $\eta$ into its unique homogeneous components $H(\eta)=$ $\sum \oplus H\left(\eta_{n}\right)$, where $\eta_{n}$ has uniform multiplicity $n$. Because of uniqueness $\eta_{n}^{g}=\eta_{n}$. Thus by irreducibility $\eta=\eta_{n}$ for some $n$.

For each finite dimensional subspace $V \subseteq N$, the restriction of $\eta$ to $V$ gives rise to a unique projection valued measure $P(V)$ on $V$ (so that $\eta(E)=\int e^{i\langle A, E\rangle} d P(A)$ ). The $P(V)$ are coherent in the sense that if $V \subseteq W$, then under that natural map $W^{\wedge} \rightarrow V^{\wedge}, P(W) \rightarrow P(V)$. Now for each vector $v \in H(\pi)$, the cylinder measure $d P_{v, v}$ has a continuous Fourier transform defined on $N$, by continuity of the representation $\eta$. Because $N$ is nuclear, this implies that $d P_{v, v}$ extends uniquely to a regular measure on $N^{\wedge}=\mathscr{D} \Lambda^{1} \otimes g$ (Minlos's theorem - see [Ge]). Thus there is a unique regular projection valued measure $P$ on $N^{\wedge}$ which projects to $P(V)$ for each $V$.

Because $P$ is homogeneous we can find the following kind of realization:

$$
\begin{aligned}
H(\eta) & =L^{2}\left(N^{\wedge}, v\right) \otimes \mathscr{H}, \\
\eta^{\prime} & =L^{\infty}(v) \otimes \mathscr{L}(\mathscr{H}), \\
\eta(E) & =\int e^{i\langle A, E\rangle} d v(A),
\end{aligned}
$$

where $\mathscr{H}$ is a separable complex Hilbert space, and the measure class of $v$ is uniquely determined.

The uniqueness of the measure class and $\eta^{g} \cong \eta$ implies that $v$ is quasi-invariant for $C^{\infty}(X, G)$. Irreducibility of $\rho$ implies ergodicity. Finally the form of the commutant $\eta^{\prime}$ implies the realization of $\pi$ in (2.4).

If we start with an irreducible unitary representation for $\hat{M}$, then we can draw a similar conclusion, although we cannot make the action as explicit. Here we will simply observe that the dual of the kernel $\mathbb{\mathbb { 1 }} \times \Lambda^{2} X \otimes \underline{g}^{\wedge}$ is identifiable with $\mathbf{Z} \times \mathscr{D} \Lambda^{1} \otimes \underline{g}$, where $C^{\infty}(X, G)$ acts by $g \cdot(k, A)=\left(k, g A g^{-\underline{1}}+k g d g^{-1}\right)$. If we consider the hyperplane $k=1$, then we should expect the appropriate measure class to be represented by "exp $\left(-\int_{X}\left|F_{A}\right|^{2}\right) D A$ ". 


\section{Section 3. The Mickelsson-Rajeev Extension and Symmetric Pairs}

Suppose $X$ is a compact spin manifold of odd dimension $d$. A Dirac operator $\not$ (possibly coupled with a G-potential) will act as an unbounded self-adjoint operator on the Hilbert space $H$ of spinors with values in $C^{n}$. Suppose $g \in C^{\infty}(X, G)$ and $g=\left(\begin{array}{ll}a & b \\ c & d\end{array}\right)$ relative to the splitting $H=H_{+} \oplus H_{-}$, where $H_{+}$corresponds to the positive part of the spectrum for $\not{\phi}$. A fundamental fact is that $b$ and $c$ belong to any of the Schatten ideals $I_{p}$ for $p>d$, but not to $I_{d}$, unless $g$ is constant ([C]). This idea is central in Connes's theory of noncommutative differential geometry and G. Segal's global approach to loop groups. Mickelsson and Rajeev exploited the same idea in developing a universal model for the Mickelsson-Faddeev extension.

In this section we will reconstruct MR's universal model along the lines of the previous section. In particular, we will mainly consider the analogue of $M$. This time we will be able to give a complete analysis of the unitary representations.

Let $g(\infty)=\underline{k}(\infty) \oplus p(\infty)$ be a Cartan decomposition for a classical infinite symmetric pair. The example of principal interest can be described as follows. Let $H=H_{+} \oplus H_{-}$, where $H_{ \pm}$are complex Hilbert spaces with orthonormal bases $\left\{\varepsilon_{j}: \pm j=1,2, \ldots\right\}$, respectively. Then $\underline{g}(\infty)=\underline{u}(2 \infty)$ consists of all skew-hermitian operators on $H$ which are finite rank relative to $\left\{\varepsilon_{j}\right\}$,

$$
\begin{aligned}
& \underline{k}(\infty)=\left\{\left(\begin{array}{ll}
\alpha & 0 \\
0 & \delta
\end{array}\right) \in \underline{u}(2 \infty)\right\}, \\
& \underline{p}(\infty)=\left\{\left(\begin{array}{ll}
0 & \beta \\
\gamma & 0
\end{array}\right) \in \underline{u}(2 \infty)\right\} .
\end{aligned}
$$

If $\underline{k}(\infty)=\underline{u}(\infty)$, we let $\underline{k}=\underline{u}(H)$, the Banach Lie algebra of all bounded skewhermitian operators on $H$; similarly if $\underline{k}(\infty)=\underline{o}(\infty), \underline{k}=\underline{o}\left(H_{\mathrm{R}}\right)$, etc. For each $1 \leqq p$ $\leqq \infty$, we let

$$
\underline{g}_{(p)}=\underline{k} \oplus \underline{p}_{p},
$$

where $\underline{p}_{p}$ denotes the completion of $\underline{p}(\infty)$ in the Schatten class $I_{p}$. The pair $\left(\underline{g}_{(p)}, k\right)$ is then a Banach symmetric pair. Note $\underline{p}_{p}$ is equipped with a non-Riemannian norm if $p \neq 2$, however.

The corresponding global objects will be denoted by $(G(\infty), K(\infty))$ and $\left(G_{(p)}, K\right)$. If $\tau$ is the Cartan involution for the pair $(G, K)$, then $G_{(p)}$ is the identity component of $\left\{g \in G: g g^{-\tau}=1+p\right.$-class $\}$ (here $(G, K)=(G L(H), O(H))$ if $(G(\infty), K(\infty))=$ $(G L(\infty), O(\infty))$, etc.) This construction can be carried through for any symmetrically normed ideal, and conjecturally these examples then exhaust all simple infinite rank symmetric spaces, up to coverings. In our example

$$
\begin{aligned}
G_{(p)} & =U_{(p)}=\left\{\left(\begin{array}{l}
a b \\
c d
\end{array}\right) \in U(H): b \in I_{p}, \quad \operatorname{index}(a)=0\right\}, \\
K & =U\left(H_{+}\right) \times U\left(H_{-}\right) .
\end{aligned}
$$

(The other compact type pairs are described explicitly in the appendix to [Pi1].) 
For $\xi, \eta \in \underline{g}_{(p)}$, let

$$
c(\xi, \eta)=2\left[\xi_{-}, \eta_{-}\right] \in \underline{k}_{p / 2},
$$

where $\xi_{-}$is the $p$-component of $\xi$, and $\underline{k}_{q}=\underline{k} \cap I_{q}$.

In the event that $p \leqq 2, c$ has values in $\underline{k}_{1}$. For the hermitian symmetric pairs we can take a trace to obtain a nontrivial ordinary $\mathbf{R}$-valued cocycle (to be precise we must take $\operatorname{tr}\left(\left.c(\xi, \eta)\right|_{H_{+}}\right)$. This is a multiple of the cocycle that arises for the standard commutation or anticommutation relations $(\operatorname{Sp}(\infty, \mathbf{R}), U(\infty))$ or $(0(2 \infty), U(\infty))$, respectively - see (6.5 of [PS]). This cocycle pulls back to a multiple of the KacMoody cocycle for loop groups.

Here we want to consider $c$ itself as a cocycle. If we consider $c$ as a cocycle for the adjoint action of $\underline{g}(\infty)$ on itself, then $c$ is trivial: if $\theta(\xi)=\xi_{-}$, then

$$
d \theta(\xi, \eta)=[\xi, \theta(\eta)]-[\eta, \theta(\xi)]-\theta([\xi, \eta])=c(\xi, \eta) .
$$

This is what we would expect based on finite dimensional experience, since $H^{2}$ of a semisimple algebra is always zero, by the Whitehead lemma.

When we enlarge the domain, however, $c$ can become nontrivial. Because $c$ has values in $\underline{k}_{q / 2}$, there is considerable flexibility in how we view $c$. To be precise we can view $c$ as a cocycle

$$
c: \underline{g}_{(q)} \times \underline{g}_{(q)} \rightarrow \underline{k}_{q / 2}+\underline{p}_{\lambda}
$$

relative to the adjoint action, for any $q / 3 \leqq \lambda \leqq q$ (these bounds arise from the constraints $\left[\underline{p}_{q}, \underline{k}_{q / 2}\right] \subseteq \underline{p}_{\lambda}$ and $\left.\left[\underline{p}_{q}, \underline{p}_{\lambda}\right] \subseteq \underline{k}_{q / 2}\right)$. We will let $\left(\mathfrak{5}_{(q), \lambda}\right.$ denote the extension defined by $c$ :

$$
0 \rightarrow \underline{k}_{q / 2}+\underline{p}_{\lambda} \rightarrow \mathfrak{G}_{(q), \lambda} \rightarrow \underline{g}_{(q)} \rightarrow 0 .
$$

This extension (for appropriate $q$ and $\lambda$ ) is the analogue of $\underline{m}$ in the preceding section. The canonical nature of $c$, from the viewpoint of symmetric space theory, may be reflected by the following

(3.3) Conjecture. $H^{2}\left(\underline{g}_{(q)} ; \underline{k}_{q / 2}+\underline{p}_{\lambda}\right)=0$ for $\lambda=q$ and $=\mathbf{R} c$ for $\lambda<q$.

Before taking up the analysis of $c$, we want to point out its relation to the theory in $[\mathrm{MR}]$.

Suppose $q=4$ and $\lambda$ is at the bottom of its range: $\lambda=\frac{4}{3}$. In the special case when $(g(\infty), \underline{k}(\infty))$ is a hermitian symmetric pair, we can define a central $\mathbf{R}$ extension of $\overline{\mathfrak{5}}=\mathbb{G}_{(4), 4 / 3}$, in precise analogy with the Kac-Moody theory, by the cocycle

$$
\begin{gathered}
\hat{c}:(\mathfrak{5} \times(\mathfrak{5} \rightarrow \mathbf{R}, \\
\hat{c}((x, \xi),(y, \eta))=\operatorname{trace}\left(\left.\left\{\left[\xi_{-}, y_{-}\right]\right\}\right|_{H_{+}}\right)
\end{gathered}
$$

(where $(x, \xi),(y, \eta) \in\left(\mathfrak{F} \cong\left(\underline{k}_{2}+p_{4 / 3}\right) \oplus \underline{g}_{(4)}\right)$. This makes sense because 4 and $\frac{4}{3}$ are conjugate exponents (this makes sense more generally for $q$ and its conjugate, provided $2 \leqq q \leqq 4$. These bounds on $q$ follow from the bounds on $\lambda$. The case $q=4$ is extreme here just as $q=2$ is extreme in the Kac-Moody case).

Now the extension defined by $\hat{c}$,

$$
0 \rightarrow \mathbf{R} \rightarrow \widehat{\mathfrak{G}} \rightarrow \mathbf{W} \rightarrow 0
$$


viewed as an extension of $g_{(4)}$, is the one appearing in [MR]. Thus the problem we must address here is whether the group $\mathscr{G}$ (constructed below) corresponding to $(\mathfrak{H}$ admits nontrivial separable projective unitary representations, where the induced infinitesimal extension is $\mathbf{6}$. (Put another way, the problem is this: $G_{(2)}$ has interesting projective representations, but they never extend to $G_{(4)}$; by adding in the extension to $G_{(4)}$, can we somehow cancel the divergences?)

To answer this question we first observe that the extension

$$
\boldsymbol{G}_{(q), \lambda} \rightarrow \underline{g}_{(q)}
$$

restricts to the semidirect product extension

$$
\underline{k} \tilde{x}\left(\underline{k}_{q / 2}+\underline{p}_{\lambda}\right) \rightarrow \underline{k} .
$$

A basic fact is that unitary representations for $K \tilde{\times} \underline{p}_{\lambda}$ extend continuously to $K \tilde{\times} \underline{p}_{2}$ and not beyond (for hermitian symmetric pairs). Thus in the context of representation theory we are constrained to take $\lambda=2$ (similarly $q \leqq 4$ ). The reason is roughly the following: if $v \in H(\pi)$ is fixed by $K$, then we obtain an equivariant map

$$
K \tilde{\times} \underline{p}_{\lambda} / K \cong \underline{p}_{\lambda} \rightarrow \mathbf{P}(H(\pi)): \quad(x, g) \rightarrow \mathbf{P}(\pi(x, g) v) ;
$$

the continuity properties of this map are sharply constrained because the essentially unique invariant Riemannian structure on $\underline{p}(\infty)$ (the completion of which is $\underline{p}_{2}$ ) must coincide with the pullback of the Fubini-Study metric.

We now state the relevant results, which are proven in [Pi2], Sect. 6.

(3.5) Proposition. Suppose $\pi$ is a separable unitary representation for $K \tilde{\times}\left(\underline{k}_{q / 2}+p_{\lambda}\right)$. Then a) either $\pi$ vanishes on $\underline{k}_{q / 2}+\underline{p}_{\lambda}$, or $q \leqq 4$ and $\lambda \leqq 2$; b) if $(\underline{g}(\infty), \underline{k}(\infty))$ is a hermitian symmetric pair, then $\pi$ extends continuously to a representation of $K \tilde{\times}\left(\underline{k}_{1}+\underline{p}_{2}\right)$.

We then have the following

(3.6) Corollary. Suppose $\mathscr{G}_{(q), \lambda}$ is a Banach Lie group with Lie algebra $\mathfrak{G}_{(q), \lambda}$. If $\mathscr{G}_{(q), \lambda}$ has a separable unitary representation $\pi$ which is nontrivial on $\exp \left(\underline{k}_{q / 2}+\underline{p}_{\lambda}\right)$, then $q \leqq 4$ and $\pi$ extends continuously to $\mathscr{G}_{(q), 2}$.

Proof. Since $K \tilde{\times}\left(\underline{k}_{q / 2}+p_{\lambda}\right)$ is simply connected (it's actually contractible), there is a map of this group onto the connected Lie subgroup of $\mathscr{G}_{(q), \lambda}$ with Lie subalgebra $\underline{k} \tilde{\times}\left(\underline{k}_{q / 2}+\underline{p}_{\lambda}\right)$. This map is a local diffeomorphism. Thus (3.6) follows from (3.5) //

Thus among the groups $\mathscr{G}_{(q), \lambda}$ (assuming they exist), from a representation viewpoint the only interesting case is $q=4, \lambda=2$.

Before stating our conclusions about the MR extension, we want to observe that there does actually exist an extension

$$
0 \rightarrow \underline{g}_{2} \rightarrow \mathscr{G}=\mathscr{G}_{(4), 2} \rightarrow G_{(4)} \rightarrow 0,
$$

which has Lie algebra $\mathfrak{\xi}_{(4), 2}$. As a manifold we have

$$
\mathscr{G} \cong \underline{g}_{2} \times G_{(4)}
$$

In these coordinates the multiplication must be of the form

$$
(\xi, g)(\eta, h)=(\omega(g, h)+A d(g) \eta, g h),
$$


where $\omega$ is a group cocycle. To motivate the formula we recall that on the Lie algebra level the infinitesimal cocycle $c$ is restricted to $g(\infty) \times g(\infty)$ was exact: $c=d \theta$, where $\theta(\xi)=\frac{1}{2}(\xi-\tau(\xi))$ and $\tau$ is the Cartan involution. For $g, h \in G(\infty)$, our formula for $\omega$ is

$$
\omega(g, h)=\delta \beta(g, h), \quad \beta(g)=\frac{1}{2} Q\left(g g^{-\tau}-1\right),
$$

where $Q$ is the orthogonal projection onto $g(\infty)$ (the condition that $g \in G_{(4)}$ means that $\left.g g^{-\tau} \in G_{4}\right)$. Note the linearization of $\beta$ is $\theta$. What we must check is that $\omega=\delta \beta$ has the requisite continuous extension to $G_{(4)} \times G_{(4)}$. This follows from the computation

$$
\begin{aligned}
2 \omega(g, h) & =2 \delta \beta(g, h)=g Q\left(h h^{-\tau}-1\right) g^{-1}-Q\left(g h h^{-\tau} g^{-\tau}-1\right)+Q\left(g g^{-\tau}-1\right) \\
& =-Q\left\{g\left(h h^{-\tau}-1\right)\left(g^{-\tau} g-1\right) g^{-1}\right\} .
\end{aligned}
$$

This should be compared with (2.3).

We now take up the MR extension. This is a topologically nontrivial extension

$$
0 \rightarrow \mathbb{T} \rightarrow \hat{\mathscr{G}}_{(4), 4 / 3} \rightarrow \mathscr{G}_{(4), 4 / 3} \rightarrow 0,
$$

corresponding to the cocycle $\hat{c}$ in (3.4). Since $\hat{c}$ vanishes on $\underline{k} \tilde{x}\left(\underline{k}_{2}+\underline{p}_{4 / 3}\right)$,

$$
K \tilde{x}\left(\underline{k}_{2}+\underline{p}_{4 / 3}\right) \rightarrow \hat{\mathscr{G}}_{(4), 4 / 3},
$$

as in the proof of (3.6). Thus (3.5) shows that any separable unitary representation of $\mathscr{G}_{(4), 4 / 3}$ will actually extend to a continuous projective representation of $\mathscr{G}_{(4), 2}$. But $\widehat{\mathscr{G}}_{(4), 4 / 3}$ does not extend to a group extension of $\mathscr{G}_{(4), 2}$. Thus

(3.9) Proposition. Every separable unitary representation of $\hat{\mathscr{G}}_{(4), 4 / 3}$ vanishes on $T$ in (3.8), and extends to an ordinary representation for $\mathscr{G}_{(4), 2}$.

At this point we cannot rule out the possibility that $\mathscr{G}_{(4), 2}$ has separable projective unitary representations. Such a representation would give rise to a central extension

$$
0 \rightarrow \mathbb{T} \mapsto \hat{\mathscr{G}}_{(4), 2} \rightarrow \mathscr{G}_{(4), 2} / L \rightarrow 0,
$$

where $L$ is a discrete $G_{(4)}$ invariant subgroup of $\underline{g}_{2}$. We can eliminate the possibility that the extension is smooth and nontrivial.

(3.11) Proposition. $H^{2}\left(\boldsymbol{6}_{(4), 2} ; \boldsymbol{R}\right)=0$.

Proof. Suppose $\omega$ represents a cohomology class. The closed condition is that $\omega(X,[Y, Z])+$ cyclic permutations $=0$. In particular

$$
\omega([(0, \eta),(x, 0)],(0, y))+\omega((x, 0),[(0, \eta),(y, 0)])=0 .
$$

The only invariant bilinear form on the simple algebra $[g(n), g(n)]$ is the Killing form, which is symmetric. Since $\bigcup_{k}[\underline{g}(n), \underline{g}(n)]$ is dense in $\underline{g}_{2}$, it follows that $\left.\omega\right|_{g_{2}}=0$.

We now consider terms of the form $\omega((0, \eta),(x, 0))$. For $\eta \in g_{(4)}$ fixed, this defines a real continuous linear functional of $x \in g_{2}$. We can identify $\underline{g}_{2}{ }_{2} \cong g_{2}$ (we will denote the pairing by $()$,$) . Thus we obtain a map \theta: \underline{g}_{(4)} \rightarrow \underline{g}_{2}$ such that

$$
\omega((0, \eta),(x, 0))=(\theta(\eta), x) .
$$

The closed condition applied to $(0, \xi),(0, \eta)$ and $(x, 0)$ implies that 


$$
\theta([\xi, \eta])=[\theta(\xi), \eta]+[\xi, \theta(\eta)]
$$

In particular $\theta: g_{2} \rightarrow g_{2}$ defines a derivation, which implies the existence of an operator $F \in g$ such that $\theta(\xi)=[F, \xi]$ (see II.5 of $[\mathrm{dH}]$ ).

Because $\bar{\theta}: \underline{g}_{(4)} \rightarrow \underline{g}_{2}$, we see that $F=F_{-} \in \underline{p}_{2}$ (for $\left[F_{+}, \underline{k}\right] \in I_{2}$ implies that $F_{+}=c J$, where $J$ defines the complex structure in the event that $(g(\infty), \underline{k}(\infty))$ is hermitian symmetric, $F_{+}=0$ otherwise; the condition $\left[F_{+}, \underline{p}_{4}\right] \subseteq I_{2}$ then implies $\left.F_{+}=0\right)$. Therefore

$$
\omega((0, \eta),(x, 0))=([F, \eta], x)=(F,[\eta, x]) .
$$

Let $\phi$ denote the linear functional defined on $\mathfrak{G}_{(4), 2} \cong \underline{g}_{2} \times \underline{g}_{(4)}$ by $F$ :

$$
\phi:(x, \xi) \rightarrow(F, x) .
$$

Now consider the equivalent representative of our cohomology class

$$
\omega^{\prime}(\cdot, \cdot)=\omega(\cdot, \cdot)-\phi([\cdot, \cdot]) .
$$

The form $\omega^{\prime}$ vanishes identically on $\underline{g}_{2}$ and on crossterms. Therefore $\omega^{\prime}$ represents a class in $H^{2}\left(g_{(4)} ; \mathbf{R}\right)$. But this space is zero. This proves (3.11)

Remark. A corollary of the proof is that $H^{2}\left(\left(_{(4), 4 / 3} ; \mathbf{R}\right)\right.$ is generated by the cocycle (3.4).

There remains the problem of determining the separable unitary representations for the group $\mathscr{G}_{(4), 2}$. The calculation in Proposition 3.21 of [Mi2] (which involves an implicit assumption of smoothness) suggests that any such representation vanishes on the kernel of the projection $\mathscr{G}_{(4), 2} \rightarrow G_{(4)}$.

Acknowledgement. I thank S. G. Rajeev for several useful conversations.

\section{References}

[dH] de la Harpe, P.: Classical Banach-Lie Algebras and Banach-Lie Groups of Operators in Hilbert Space. Lecture Notes in Mathematics, vol. 285, Berlin, Heidelberg, New York: Springer 1972

[Mi1] Mickelsson, J.: Kac-Moody groups and the Dirac determinant line bundle. In: Topological and geometrical methods in field theory, p. 117-131. Hietarinta, J., Westerholms, J. (eds.), Singapore: World Scientific 1986

[Mi2] Mickelsson, J.: Current algebra representation for the $3+1$ dimensional Dirac-Yang-Mills theory. Commun. Math. Phys. 117, 261-277 (1988)

[MR] Mickelsson, J., Rajeev, S. G.: Current algebras in $d+1$ dimensions and determinant bundles over infinite dimensional Grassmannians. Commun. Math. Phys. 116, 365-400 (1988)

[Pi1] Pickrell, D.: Separable representations for automorphism groups of infinite rank symmetric spaces, to appear in J.F.A.

[Pi2] Pickrell, D.: Mackey analysis of infinite semidirect products, submitted to Pac. J. Math.

[PS] Pressley, A., and Segal, G.: Loop groups. Oxford: Oxford University Press 1987

[Se] Segal, G.: Faddeev's anomaly in Gauss's law, preprint

[c] Connes, A.: Non-commutative differential geometry. Publ. Math. I.H.E.S. 62, 257-360 (1986)

[Fr] Frenkel, I.: Beyond Affine Lie Algebras. Proceedings of the I.C.M., 1986, p. 821

[Ge] Gelfand, I., Vilenkin, N.: Generalized functions, vol. 4. New York: Academic Press 1964

Communicated by A. Jaffe

Received October 20, 1988 
